\title{
Prospects of higher-order Laguerre Gauss modes in future gravitational wave detectors
}

\author{
Simon Chelkowski, ${ }^{1}$ Stefan Hild, ${ }^{1}$ and Andreas Freise ${ }^{1}$ \\ ${ }^{1}$ School of Physics and Astronomy, University of Birmingham, Edgbaston, Birmingham B15 2TT, UK
}

(Dated: August 22, 2018)

\begin{abstract}
The application of higher-order Laguerre Gauss (LG) modes in large-scale gravitational wave detectors has recently been proposed. In comparison to the fundamental mode, some higher-order Laguerre Gauss modes can significantly reduce the contribution of coating Brownian noise. Using frequency domain simulations we give a detailed analysis of the longitudinal and angular control signals derived with a $\mathrm{LG}_{33}$ mode in comparison to the fundamental $\mathrm{TEM}_{00}$ mode. The performance regarding interferometric sensing and control of the $\mathrm{LG}_{33}$ mode is found to be similar, if not even better in all aspects of interest. In addition, we evaluate the sensitivity gain of the implementation of $\mathrm{LG}_{33}$ modes into the Advanced Virgo instrument. Our analysis shows that the application of the $\mathrm{LG}_{33}$ mode results in a broadband improvement of the Advanced Virgo sensitivity, increasing the potential detection rate of binary neutron star inspirals by a factor 2.1 .

PACS numbers: $04.80 . \mathrm{Nn}, 95.75 . \mathrm{Kk}, 42.25 . \mathrm{Bs}, 95.55 . \mathrm{Ym}$
\end{abstract}

\section{INTRODUCTION}

The search for gravitational waves (GW) has led to a new class of extremely sensitive laser interferometers. The first generation of large-scale laser-interferometric gravitational wave detectors [1, 2, 3, 4] is now in operation with the aim of accomplishing the first direct detection of gravitational waves. The detector performance is limited by several fundamental and technical noises. In a constant effort the noise contributions are minimised to improve the detectors signal-to-noise ratio. One of the limiting noise sources of the currently planned second generation gravitational wave detectors will be thermal noise [5] of the mirror test masses. There exist several components to thermal noise of which the Brownian thermal noise is largest in current interferometer topologies utilising arm cavities. Cooling of the mirror test masses as currently studied in CLIO [6] reduces the thermal noise provided an appropriate material is chosen for the optics. A different way to lower the thermal noise is to change the mode shape of the laser beam inside the interferometer. All current detectors use the fundamental $\mathrm{TEM}_{00}$ mode, but several other mode shapes such as Mesa beams 7, conical modes 8 and higher-order Laguerre Gauss (LG) modes [9] have been proposed for reducing thermal noise. The basic idea is to reduce thermal noise by generating a more uniform light intensity distribution on the mirrors without introducing higher clipping losses 9 .

The proposed candidates for different beam shapes can be divided in two groups. The first group, which comprises flat-top and conical beams, would require the use of non spherical mirror shapes. As a result these modes are not compatible with current GW detectors and their spherical mirrors. Currently it is not clear to what precision these non-spherical mirrors can be manufactured and little experience in using such mirrors has been gained so far. The second group consists of higherorder Laguerre Gauss modes, which are fully compati- ble with spherical mirrors as the currently used $\mathrm{TEM}_{00}$ mode 41. So far LG modes have been mainly employed in the field of cold atom and quantum optics for example as optical tweezers [10] or waveguides [11].

Currently several techniques for the generation of higher-order LG-modes exist e.g. using holograms [12, 13], gratings 14 and mode transformers [15, 16. With these techniques a conversion efficiency of $60 \%$ [14 has been demonstrated. Recently the creation of higherorder LG-modes with a very high mode purity 17 has been reported. Our paper assumes that using these techniques, higher-order LG-modes can be created with high power output and high mode purity required in the field of GW detection. We analyse the compatibility of such higher-order LG-modes with the core interferometer in future GW detectors, using Advanced Virgo in particular as an example for a second-generation GW detector. In Section 【I we introduce the definition of the LGmodes, how they can be described in a Hermite Gauss mode basis system and how the coating brownian thermal noise is calculated depending on which LG-mode is used. Moreover we introduce some practical considerations concerning clipping loss, beam sizes and radius of curvature (RoC) of the mirrors which are essential for the later analysis. In Section III we perform a phase coupling analysis of a single arm cavity and a Michelson interferometer using higher-order LG modes in comparison to the currently used fundamental mode. We determine any differences in their phase coupling between the different longitudinal and alignment degrees of freedom. In Section IV a numerical model based on a set of Advanced Virgo design parameters is used to analyse the prospects of higher-order LG modes in comparison to the currently proposed use of the fundamental mode. The detector sensitivities of the different interferometer configurations are computed to derive the envisioned detector inspiral ranges. In total we analyse and compare three different cases with each other. 


\section{INITIAL CONSIDERATIONS}

\section{A. Hermite Gauss and Laguerre Gauss modes}

The Hermite Gauss (HG) modes and Laguerre Gauss modes both present complete basis sets such that each LG mode can be presented by a sum of HG modes and vice versa. The so-called helical Laguerre Gauss modes can be written as [18, 19]:

$$
\begin{array}{r}
u_{p, l} \quad(r, \phi, z)=\frac{1}{w(z)} \sqrt{\frac{2 p !}{\pi(|l|+p) !}} \exp (\mathrm{i}(2 p+|l|+1) \Psi(z)) \\
\times\left(\frac{\sqrt{2} r}{w(z)}\right)^{|l|} L_{p}^{l}\left(\frac{2 r^{2}}{w(z)^{2}}\right) \exp \left(-\mathrm{i} k \frac{r^{2}}{2 q(z)}+\mathrm{i} l \phi\right)
\end{array}
$$

with $r, \phi$ and $z$ as the cylindrical coordinates around the optical axis, $w(z)$ the beam radius, $\Psi(z)$ the Gouy phase, $q(z)$ the Gaussian beam parameter and $L_{p}^{l}(x)$ the associated Laguerre polynomials. The indices must obey the following relations: $0 \leq|l| \leq p$ where $p$ is the radial mode index and $l$ the azimuthal mode index.

The decomposition of these modes into Hermite Gauss modes can be performed as follows [20]:

$$
u_{p, l}(x, y, z)=\sum_{k=0}^{(2 p+1)} \mathrm{i}^{k} b(l+p, p, k) u_{2 p+l-k, k}^{H G}(x, y, z)
$$

with real coefficients

$$
\begin{aligned}
b(l+p, p, k)= & \sqrt{\frac{(2 p+l-k) ! k !}{2^{(2 p+l)}(l+p) ! p !}} \\
& \times(-2)^{k} P_{k}^{l+p-k, p-k}(0)
\end{aligned}
$$

where $P_{n}^{\alpha, \beta}(x)$ denotes the Jacobi Polynomials. It is interesting to note in Equation 2 that a given $\mathrm{LG}_{p l}$ mode is constructed of $2 p+l+1 \mathrm{HG}_{n m}$ modes of the order $n+m=2 p+l$. For example the $\mathrm{LG}_{33}$ mode is constructed out of ten Hermite Gauss modes of the order nine.

\section{B. Coating brownian thermal noise of Laguerre-Gauss modes}

According to 21] the power spectral density of displacement equivalent thermal noise is generally given by

$$
S_{x}(f)=\frac{4 k_{B} T}{\pi f} \phi U
$$

with $\phi$ being the loss angle and U the strain energy of the static pressure profile on the mirror surface normalised to $1 \mathrm{~N}$. The interested reader is referred to [22] where detailed calculations of various thermal noises such as substrate brownian, coating brownian and thermoelastic thermal noise are presented. The currently most limiting thermal noise in GW detectors is the coating brownian thermal noise. In the case of a semi-infinite mirror the coating brownian thermal noise induced by a $\mathrm{LG}_{p l}$ mode can be calculated using the strain energy

$$
U_{p, l, \text { coating }}=\delta_{c} \frac{(1+\sigma)(1-2 \sigma)}{\pi Y w^{2}} g_{p, l} .
$$

Here $\delta_{c}$ is the thickness of the coating, $\sigma$ is the Poisson ratio, $Y$ is the Young modulus, $w$ is the beam width at the mirror and $g_{p, l}$ is a scaling factor depending on the used $\mathrm{LG}_{p l}$ mode. In the case of the fundamental $\mathrm{LG}_{00}$ mode this scaling factor is $g_{0,0}=1$, whereas for a $\mathrm{LG}_{33}$ mode $g_{3,3}=0.14$ has to be used. Hence the power spectral density of displacement equivalent coating brownian thermal noise is more than a factor of seven smaller for a $\mathrm{LG}_{33}$ mode in comparison to the fundamental $\mathrm{LG}_{00}$ mode. For finite mirror sizes 22 finds that the deviation to the semi-infinite case are very small if the clipping loss of the beam on the mirror surface are small.

\section{Clipping loss and beam scaling factors}

For our later analysis it is important to know the clipping loss $l_{\text {clip }}$ that affects the propagating Gaussian mode at the mirror due to its finite size. It is given by

$$
\begin{aligned}
l_{\operatorname{clip}}(w, \rho, z)=1 & -\int_{0}^{2 \pi} d \phi \int_{0}^{\rho} d r \cdot r \\
& \times u(w, r, \phi, z) u^{*}(w, r, \phi, z),
\end{aligned}
$$

were $w$ is the beam radius at the mirror, $\rho$ is the radius of the mirror coating and $u(w, r, \phi, z)$ is the transversal field distribution of the mode of interest. Please note that the parameter beam radius $w$ is a measure of the beam size of the fundamental Gaussian mode $\left(\mathrm{LG}_{00}\right.$ or $\mathrm{HG}_{00}$ ). Higher-order LG or HG modes of the same beam radius actually are more spatially extended, in the sense that a significant amount light power can be detected at distances off the optical axis larger than the beam radius. Using the general definitions of the transversal field distribution for Hermite Gauss and Laguerre Gauss modes $u$ we can compute the clipping losses of any of these modes.

In Figure 1 the clipping losses for the fundamental and two higher-order LG modes are plotted over the mirrorradius to beam-radius ratio. One can see clearly that in comparison to the fundamental $\mathrm{LG}_{00}$ mode, the higherorder LG modes have a much more widely spread intensity distribution for a given beam radius. Hence they require either larger mirrors, or reduced beam radii for a fixed mirror size. Table I comprises the respective scaling factors, normalised to a optimised mirror size for a $\mathrm{LG}_{00}-$ mode with a clipping loss of $1 \mathrm{ppm}$.

One consequence of this more widely spread intensity distribution of the higher-order LG modes is of major importance for the later analysis: in order to fit a higher-order mode optimally on the same mirror as the fundamental mode, the beam radius of the higher-order mode must be different from that of the fundamental mode. This 


\begin{tabular}{l|c|c|c} 
& $\mathrm{LG}_{00}$ & $\mathrm{LG}_{33}$ & $\mathrm{LG}_{55}$ \\
\hline \hline mirror to beam radius ratio & 2.63 & 4.31 & 5.05 \\
relative mirror radius & 1 & 1.64 & 1.92 \\
relative beam radius & 1 & 0.61 & 0.52
\end{tabular}

Table I: Comparison of the mirror to beam radius ratio for 1 ppm clipping loss and the corresponding scaling factors for the beam radius and mirror radius to keep the clipping loss constant normalised to the $\mathrm{LG}_{00}$-mode with a clipping loss of $1 \mathrm{ppm}$.

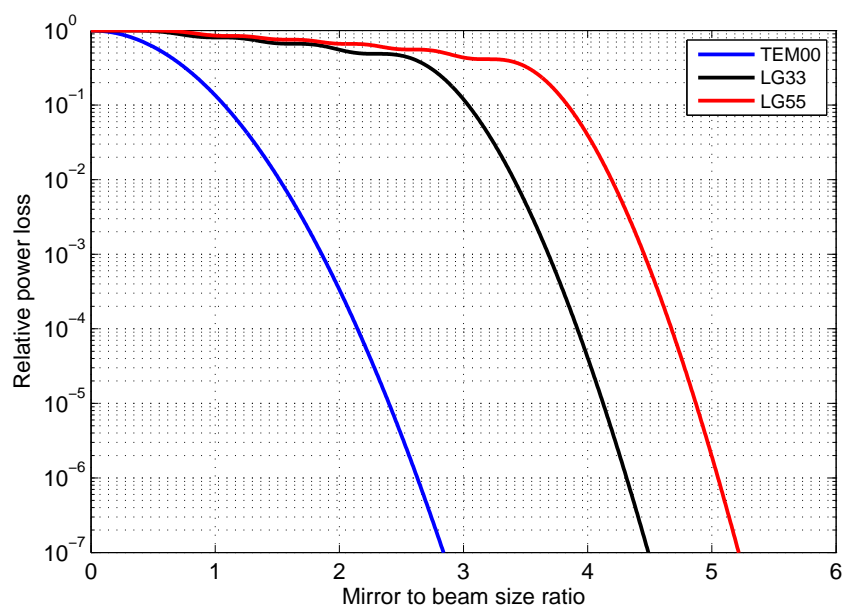

Figure 1: The plot shows the relative power loss in reflection of a finite sized mirror due to clipping loss for three different incident transversal modes over the mirror to beam radius ratio. The different curves shown are: blue curve $=\mathrm{TEM}_{00}$ mode; black curve $=\mathrm{LG}_{33}$ mode; red curve $=\mathrm{LG}_{55}$ mode. The mirror to beam radius ratio for a fixed clipping loss of $1 \mathrm{ppm}$ is given in Table I

corresponds to a different wave front curvature and consequently to a different spherical curvature of the cavity mirrors [18]. Thus changing an existing optical experiment such as an interferometer from a configuration using e.g. the $\mathrm{TEM}_{00}$ mode to a configuration using the $\mathrm{LG}_{33}$ mode, the radii of curvature of the mirrors must be changed, if one wants to keep the clipping losses at a constant level. In most cases this would necessitate in a complete exchange of the mirrors.

\section{PHASE COUPLING COMPARISON OF THE LG 33 MODE WITH THE FUNDAMENTAL HG $_{00}$ MODE}

The sensitivity of future gravitational wave detectors will be limited partly by thermal noise. The use of higherorder LG modes represents a very interesting option for reducing this limit. For a successful implementation, however, higher-order LG modes must comply with the stringent phase noise requirements in these detectors. In the following we compare the phase noise performance of the currently used $\mathrm{HG}_{00}$ mode with that of a $\mathrm{LG}_{33}$ mode which serves as a representative of the family of higherorder LG modes. The analysis was performed with the numerical interferometer simulation Finesse [23, which uses the Hermite Gauss modal expansion for describing the spatial properties of light fields transverse to the optical axis. In order to simulate higher-order LG modes we used the decomposition of higher-order LG into HG modes presented in Section III. The results of the analysis described in this section are in principle applicable to many other Laguerre Gauss modes of interest. For instance for the $\mathrm{LG}_{55}$ mode, the corresponding mirror and beam radius scaling values are given in table [.

\section{A. Configurations of interest}

Our phase noise coupling analysis uses the currently planned $3 \mathrm{~km}$ long Advanced Virgo interferometer as testbed. We compare the use of a $\mathrm{LG}_{33}$ mode with two different configurations using a fundamental mode:

1.) The $\mathrm{LG}_{00}^{\text {small }}$ configuration: This configuration uses the optical parameters presented in [24] and represents our reference configuration. The configuration uses arm cavity mirrors with RoCs $R_{C}=$ $\pm 1910 \mathrm{~m}$ which corresponds to a beam size of the fundamental $\mathrm{LG}_{00}$ mode of $w=35.2 \mathrm{~mm}$ at the mirrors surface and a corresponding waist size of

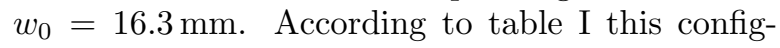
uration would have a clipping loss of $1 \mathrm{ppm}$ for a mirror radius of $\rho_{\text {small }}=92.5 \mathrm{~mm}$.

2.) The $L_{33}$ configuration: This configuration uses the higher-order Laguerre Gauss mode $\mathrm{LG}_{33}$. It shares its beam parameters with the reference LG $_{00}^{\text {small }}$ configuration to simplify the comparison. The mirror radius has to be adapted for this configuration because of the more wider intensity profile of the $\mathrm{LG}_{33}$ mode. A mirror radius of $\rho_{\text {large }}=$ $151.8 \mathrm{~mm}$ is required to maintain a clipping loss of $1 \mathrm{ppm}$ (see Table I).

3.) The $L G_{00}^{\text {large }}$ configuration: The third configuration uses the fundamental $\mathrm{LG}_{00}$ mode in combination with the larger mirrors with radius $\rho_{\text {large }}$ used in the $\mathrm{LG}_{33}$ configuration. As a result the beam size on the mirrors can be increased to $w=57.7 \mathrm{~mm}$ while still maintaining a clipping loss of $1 \mathrm{ppm}$. Hence the waist size decreases to $w_{0}=8.9 \mathrm{~mm}$ and all other beam parameters change accordingly.

The beam parameters of each configuration are displayed in Table I and their transversal intensity distribution on the mirror surface is shown in Figure 2, It is worth noting, that a comparison between the $\mathrm{LG}_{33}$ and the $\mathrm{LG}_{00}^{\text {large }}$ configuration is much more reasonable because these two configurations use the same mirror sizes. 

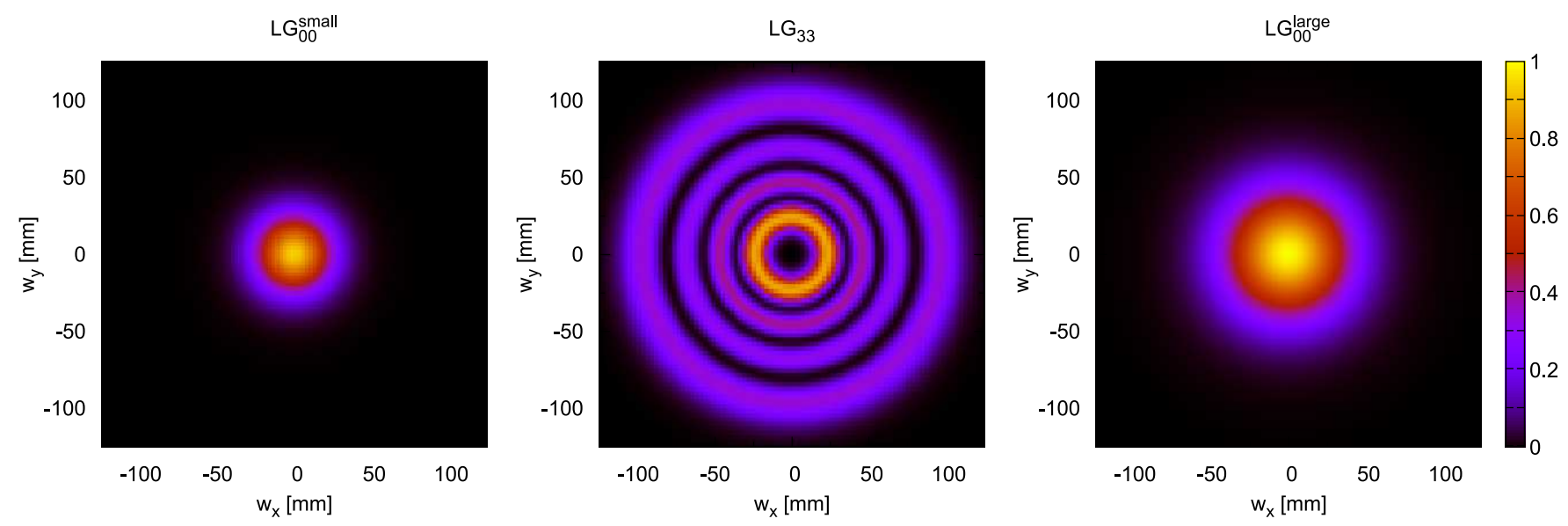

Figure 2: Transversal intensity distribution at the mirror surface for the three configurations under investigation.

\begin{tabular}{l|c|c|c} 
& $\mathrm{LG}_{00}^{\text {small }}$ & $\mathrm{LG}_{33}$ & $\mathrm{LG}_{00}^{\text {large }}$ \\
\hline \hline$R_{C}[\mathrm{~m}]$ & 1910 & 1910 & 1536.7 \\
$w[\mathrm{~mm}]$ & 35.2 & 35.2 & 57.7 \\
$w_{0}[\mathrm{~mm}]$ & 16.3 & 16.3 & 8.9
\end{tabular}

Table II: Beam and mirror parameters of the three different configurations used in the analysis. There exist always two RoC settings for achieving a given spot size on the mirrors. We have chosen the cavity geometry which reduces the radiation pressure induced alignment instabilities [25].

\section{B. Tilt to longitudinal phase coupling of a single cavity}

The first part of our analysis is performed for a single cavity. At first we want to investigate the longitudinal error signal of each configuration in order to find out how they compare against each other. To generate this longitudinal error signal we use the Pound-Drever-Hall technique based on a modulation/demodulation scheme [26]. We found that all resulting error signals are identical, which confirms that such error signals only depend on the average phase of the beam, and is independent of its modal distribution.

Next we analyse the coupling of misalignment of a cavity into longitudinal phase noise for the three configurations of interest. The first results are comprised in Figure 3 which shows the intra cavity power over the tilt angle $\beta$ of the end mirror (EMX) and the longitudinal tuning $\phi$ of the cavity for each configuration. The tuning value $\phi$ of the cavity is the result of a modulo division of the cavity length by the wavelength. In the following this tuning is given in degrees with $360^{\circ}$ referring to one wavelength:

$$
\phi=360^{\circ} \cdot(L \bmod \lambda)
$$

In the analysis the tilt angle $\beta$ of the cavity's end mirror - shown on the $\mathrm{x}$-axis — was varied between $0 \mu \mathrm{rad}$ to
$1 \mu \mathrm{rad}$. On the $\mathrm{y}$-axis the tuning $\phi$ was chosen such that the resonance of the cavity is clearly visible. One can see that a tilt of the end mirror changes the tuning of the resonance for every configuration. Because the tuning refers to the length of the cavity, there is indeed a coupling from tilt into the longitudinal phase degree of freedom of the cavity. The coupling strength is different for each configuration. Nevertheless, configuration $\mathrm{LG}_{00}^{\text {small }}$ and $\mathrm{LG}_{33}$ behave in a very similar manner. Both show a shift of the resonance condition of approximately $\Delta \phi=0.4^{\circ}$ for a tilt of $1 \mu \mathrm{rad}$. In contrast, configuration $\mathrm{LG}_{00}^{\text {large }}$ shows an increased coupling strength by more than one order of magnitude. For a tilt of $1 \mu \mathrm{rad}$ the cavity tuning of for the $\mathrm{LG}_{00}^{\text {large }}$ configuration changes by $\Delta \phi \approx 5^{\circ}$.

The tilt of the end mirror (EMX) also changes the geometry of the eigenmode of the cavity. Compared to the input beam the eigenmode of the cavity is tilted as well. As a result the mode matching efficiency into the cavity is decreased, leading to a reduced intra cavity power. This behaviour is hardly visible in Figure 3 but clearly shown in Figure 4 . The $\mathrm{LG}_{00}^{\text {small }}$ configuration is the most robust in terms of decreased intra cavity power, followed by the $\mathrm{LG}_{33}$ and the $\mathrm{LG}_{00}^{\text {large }}$ configurations.

In conclusion of this section the $\mathrm{LG}_{00}^{\text {small }}$ configuration performs best in all aspects of the analysis. Nevertheless as stated initially a comparison between the other two configurations is much fairer because they share the same mirror size. Taking this into account, the favourable mode is the $\mathrm{LG}_{33}$, because its tilt induced coupling into the longitudinal phase and into the intra cavity power is much less than for a $\mathrm{LG}_{00}$ mode on the same mirrors.

\section{Alignment analysis of a single arm cavity}

The proper sensing and control of the alignment degrees of freedom of a gravitational wave detector is critical for its successful operation. Hence, a comparison analysis of the alignment error signals of the individual arm cavities for the three different transversal mode con- 

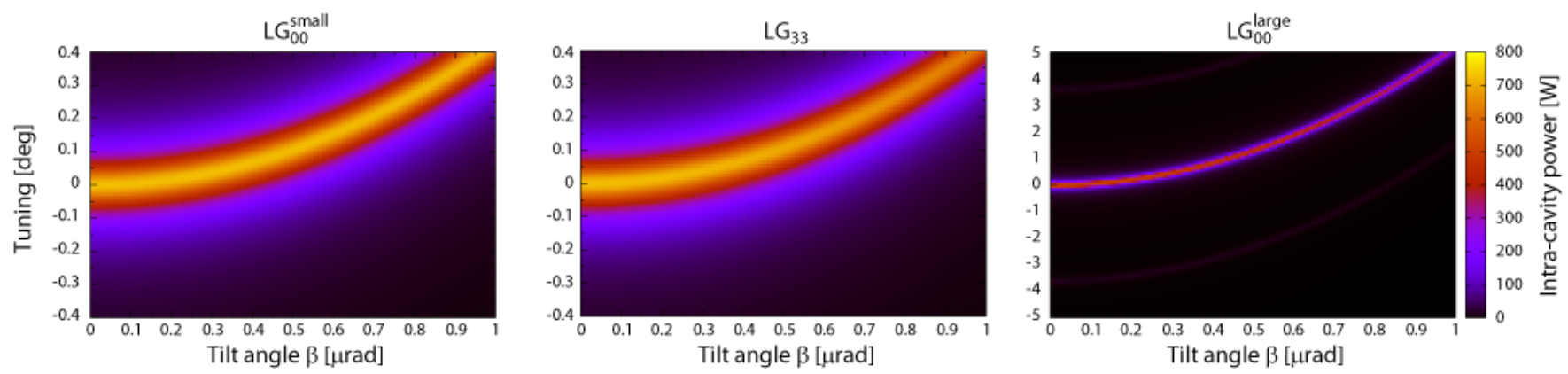

Figure 3: Intra cavity power over tilt angle $\beta$ of the end mirror (EMX) and longitudinal tuning $\phi$ of a single cavity shown for all three configurations of interest. The first two configurations show almost the same coupling from tilt into longitudinal tuning which is more than an order of magnitude lower than the coupling of the third configuration.

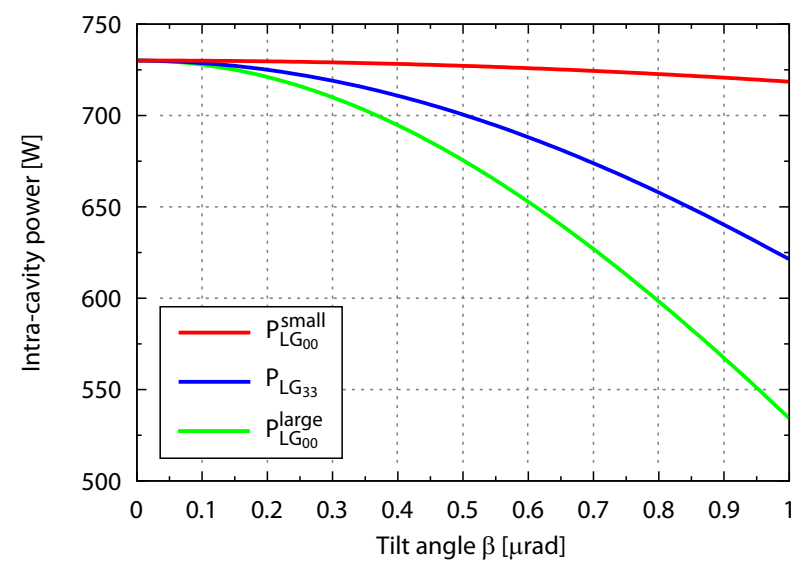

Figure 4: Comparison between all three cases and their coupling between tilt of mirror EMX to the tuning of mirror IMX.

figurations defined in Section IIIA is needed and presented in this section. Full alignment control systems of advanced GW detectors are very complex and depend on the details of the detector design. The concept however, is firmly based on the control of resonant cavities and we can use a single Fabry Perot cavity to test whether LG modes are compatible with current alignment control systems. The analysis uses an optical layout as shown in Figure 5 (A). An electro-optic modulator (EOM) imprinting a phase modulation with frequency $\Omega$ in combination with two quadrant photodiodes is used to generate alignment error signals for the two arm cavity mirrors using the Ward technique described in [27. Here each quadrant photodiode is responsible for obtaining an alignment error signal of one arm cavity mirror. In the following we only consider mirror rotations around vertical axis. The results however remain applicable for the tilt degree of freedom of each mirror. The optimisation of the error signals does not use the theoretical optimal parameters but is done by tuning the parameters; this reflects realistic experimental procedures. The two quadrant photodiodes are placed such that their Gouy phase difference is $90^{\circ}$ leaving the total Gouy phase arbitrary. Each photo- diode current is then demodulated at frequency $\Omega$. The demodulation phase is chosen to maximise the error signal slope for the corresponding mirror. An example of the corresponding alignment error signals for the misalignment of both arm cavity mirrors using the $\mathrm{LG}_{00}^{\text {small }}$ configuration can be seen in Figure 6. A good way of comparing different alignment error signals is looking at the resulting control matrix [28, 29]. In our example the control matrix contains the values of the alignment error signal slopes $\sigma$ in the working point generated by the two quadrant photodiodes QPD1 and QPD2 for a misalignment of the arm cavity mirrors IMX and EMX, see Figure 5(A). The subscript of $\sigma$ indicates the readout diode and the superscript refers to the mirror of interest. Hence, the control matrix is given in general by the following expression

$$
C_{\text {configuration }}=\left(\begin{array}{cc}
\sigma_{\mathrm{QPD} 1}^{\mathrm{IMX}} & \sigma_{\mathrm{QPD} 1}^{\mathrm{EMX}} \\
\sigma_{\mathrm{QPD} 2}^{\mathrm{IMX}} & \sigma_{\mathrm{QPD} 2}^{\mathrm{EMX}}
\end{array}\right) .
$$

Applying the optimisation procedure for the alignment signals described earlier for all three configurations of interest, results in the following three control matrices.

$$
\begin{aligned}
C_{\mathrm{LG}_{00}^{\text {small }}} & =\left(\begin{array}{ll}
5.6152 & 0.0477 \\
2.1607 & 3.5878
\end{array}\right) \\
& =5.6152\left(\begin{array}{cc}
1 & 0.009 \\
0.385 & 0.639
\end{array}\right) \\
C_{\mathrm{LG}_{33}} & =\left(\begin{array}{ll}
7.444 & 0.022 \\
2.741 & 4.771
\end{array}\right) \\
& =7.444\left(\begin{array}{cc}
1 & 0.003 \\
0.368 & 0.641
\end{array}\right) \\
C_{\mathrm{LG}_{00}^{\text {large }}} & =\left(\begin{array}{cc}
17.774 & 15.330 \\
11.472 & 2.725
\end{array}\right) \\
& =17.774\left(\begin{array}{cc}
1 & 0.862 \\
0.645 & 0.153
\end{array}\right)
\end{aligned}
$$

For an ideal control matrix all matrix elements on the diagonal would be one and the off-diagonal elements are zero. Comparing the resulting control matrices, one can see that the $\mathrm{LG}_{00}^{\text {large }}$ configuration performs worse than 


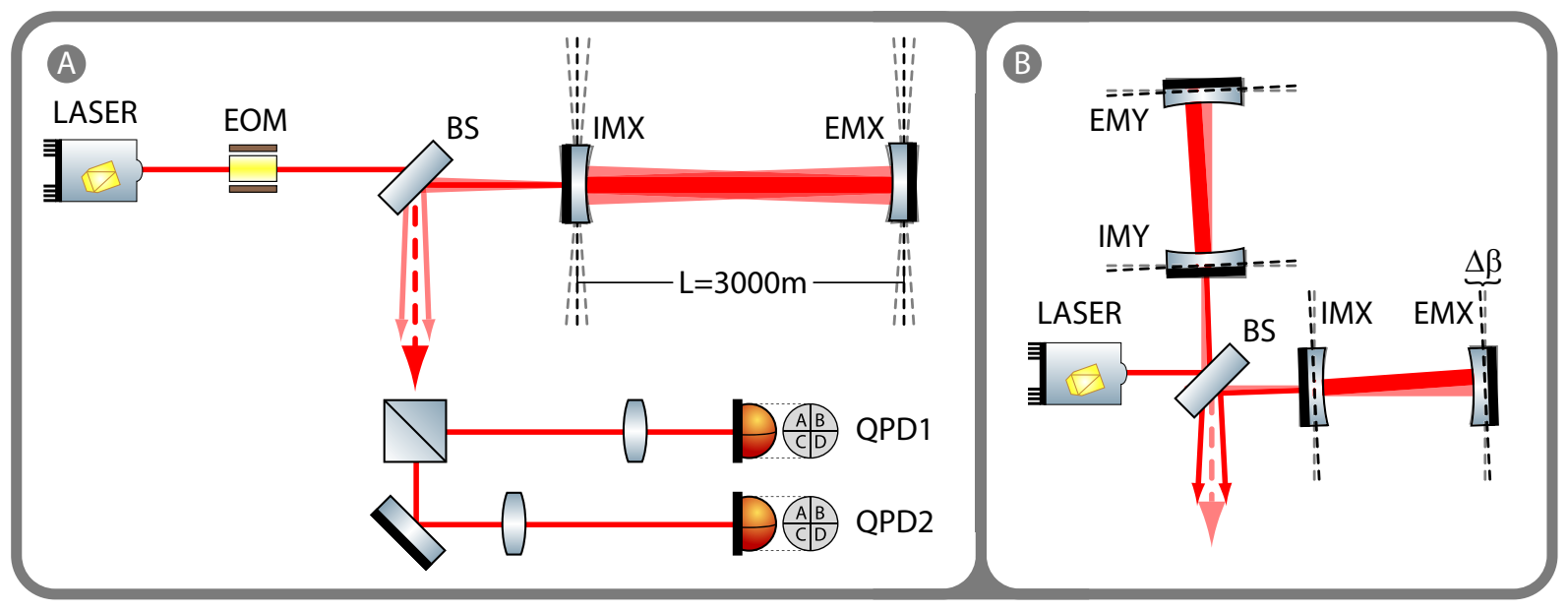

Figure 5: Different optical layouts used in the alignment analysis described in Section III C. Left (A): The generation of alignment error signals for a single arm cavity. Right (B): Michelson interferometer with differentially misaligned arm cavities to study the power coupling into the output port of the interferometer.

the other two: both mirrors couple much more strongly into QPD1 than into QPD2 making the alignment error signals far from ideal. The $\mathrm{LG}_{00}^{\text {small }}$ and the $\mathrm{LG}_{33}$ configuration show a much better and almost even performance. This is represented by the fact that in both of these configurations the misalignment of mirror IMX couples three times more strongly into QPD1 than into QPD2. Any misalignment of mirror EMX couples a factor of 75 stronger into QPD2 compared to the signal sensed with QPD1 for the $\mathrm{LG}_{00}^{\text {small }}$ configuration. This factor increases further to 216 in the case of the $\mathrm{LG}_{33}$ configuration.

In conclusion we can say that the $\mathrm{LG}_{00}^{\text {large }}$ is outperformed by the other two. The reason for this is not to be found in the mode shape but in the cavity geometry. The RoCs of the mirrors of the $\mathrm{LG}_{00}^{\text {small }}$ and the $\mathrm{LG}_{33}$ configurations are the same, which results in almost the same control matrix. In contrast the RoCs of the mirrors of the $\mathrm{LG}_{00}^{\text {large }}$ configuration are much smaller. For the $\mathrm{LG}_{00}^{\text {large }}$ configuration one obtains a cavity gparameter [18] of $g=0.91$ which is very close to the instability border of unity. The other two configurations have a g-factor of 0.33 which corresponds to a much more stable and robust geometry.

\section{Coupling of differential arm cavity misalignment into the output port power}

An important measure for the performance of the different optical modes in a full interferometer configuration is how much the differential misalignment of the arm cavities and the corresponding position change of each cavity's eigenmode couples with the power in the interferometers output port due to the reduce mode overlap on the beam splitter. A sketch of this behaviour can be seen in Figure 5 (B). This coupling mechanism can generate a signal which is indistinguishable from a $\mathrm{GW}$ signal. Hence it is important to analyse how higher-order Laguerre Gauss modes compete with the currently used fundamental mode. The following analysis compares this coupling mechanism for the three configurations of interest with respect to a reference value. This reference value is the limit Advanced LIGO 30] specifies for the differential arm length deviation which is supposed to be smaller than $10^{-15} \mathrm{~m} 31$. This deviation together with the envisaged dark fringe offset of $10^{-12} \mathrm{~m} \mathrm{[32}$ allows us to calculate the expected increase in power in the output port of the interferometer to be $7.124 \cdot 10^{-9} \mathrm{~W}$. This reference value can now be compared to the output power enhancement of the three configurations of interest for a misalignment of up to $200 \mathrm{prad}$ (see Figure 7). The $\mathrm{LG}_{00}^{\text {small }}$ configuration shows the smallest coupling from differential tilt of the arm cavities into the output port power followed by the $\mathrm{LG}_{33}$ and finally the $\mathrm{LG}_{00}^{\text {large }}$ configuration. The values where each configuration crosses the reference limit of $7.124 \cdot 10^{-9} \mathrm{~W}$ are summarised in Table ஹ,

\begin{tabular}{l|c|c} 
& misalignment angle $\beta$ & coupling scaling factor \\
\hline \hline $\mathrm{LG}_{00}^{\text {small }}$ & 148 prad & 1 \\
$\mathrm{LG}_{33}$ & $46 \mathrm{prad}$ & 3.2 \\
$\mathrm{LG}_{00}^{\text {large }}$ & $26 \mathrm{prad}$ & 5.7
\end{tabular}

Table III: Results of the coupling analysis between the differential arm cavity misalignment and the interferometers output port power. For each of the three mode configurations of interest the misalignment angle is given at which the interferometer output power has risen from it nominal value of $6.412 \cdot 10^{-9} \mathrm{~W}$ to the reference limit of $7.124 \cdot 10^{-9} \mathrm{~W}$. The given coupling scaling factor shows the relative coupling strength of each configuration referenced to the $\mathrm{LG}_{00}^{\text {small }}$ configuration.

In conclusion the $\mathrm{LG}_{00}^{\text {small }}$ configuration performs at 

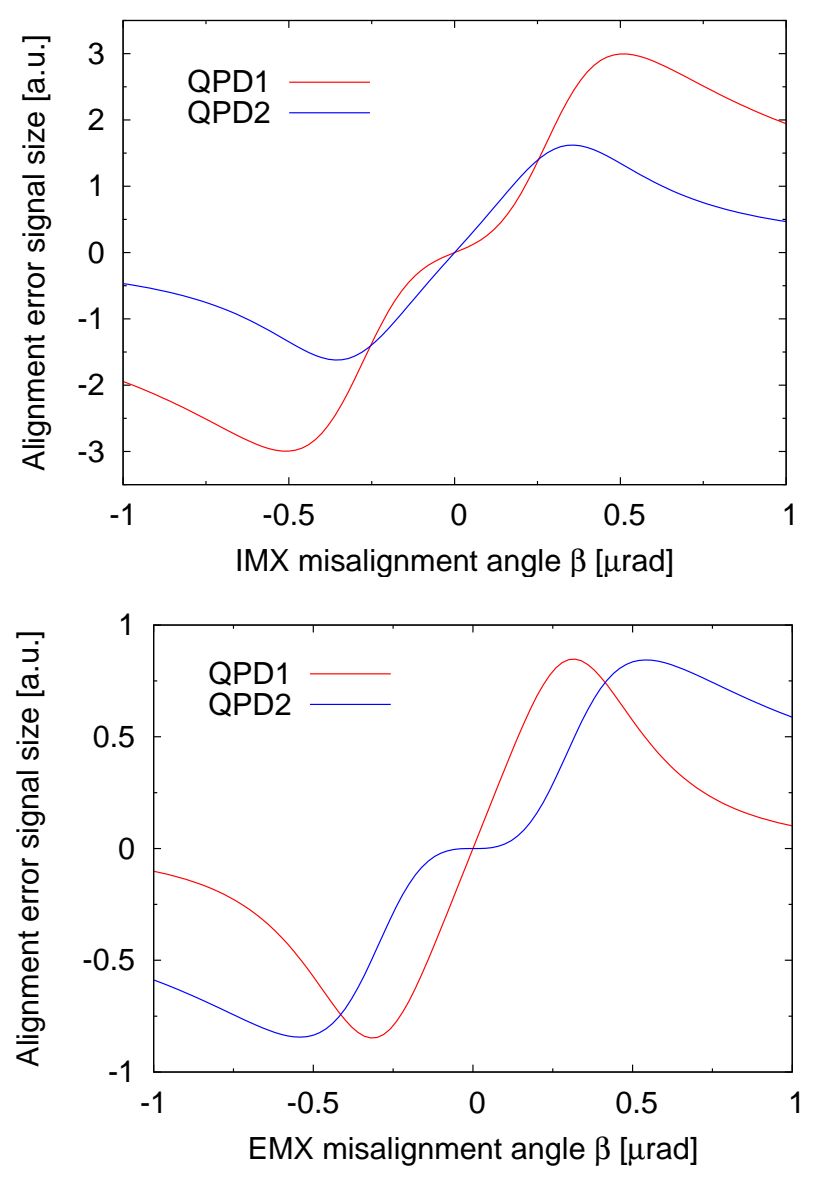

Figure 6: Comparison of the obtained alignment error signals in the $\mathrm{LG}_{00}^{\text {small }}$ case sensed with the two quadrant photodiodes while mirror IMX (top) and mirror EMX (bottom) is misaligned.

least a factor of 3.2 better than the other two configurations. Nevertheless if we compare the two configurations which use the same mirror size, we find that the $\mathrm{LG}_{33}$ performes much better than the $\mathrm{LG}_{00}^{\text {large }}$ configuration.

Overall we can conclude from the performed phase coupling comparison analysis that higher-order Laguerre Gauss modes are suitable for the implementation in future GW detectors. The direct comparison between the two configurations which use the same mirror sizes - the $\mathrm{LG}_{00}^{\text {large }}$ and the $\mathrm{LG}_{33}$ configuration - clearly shows that in all of the presented phase coupling analyses the configuration using higher-order Laguerre Gauss modes performs better than the configuration based on the traditionally used fundamental mode. This serves to underline the great potential and prospects of using higher-order Laguerre Gauss modes in future GW detectors.

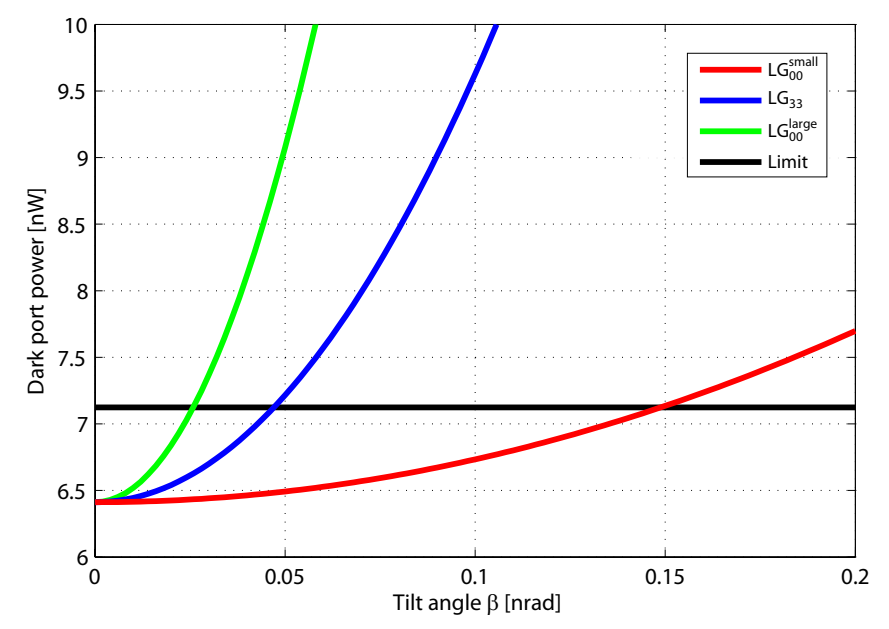

Figure 7: Comparison of the dark port power when the arm cavities are differentially misaligned (see Fig. 5 (B) in reference to the dark fringe power that results from a differential arm length deviation of $10^{-15} \mathrm{~m}$ while the interferometers dark fringe offset is $10^{-12} \mathrm{~m}$.

\section{PROSPECTS FOR ADVANCED VIRGO}

In this section we focus on the currently planned second generation GW detector Advanced Virgo 33] and its sensitivity. The design efforts for Advanced Virgo are rapidly progressing, yielding frequently improved detector configurations. Hence the configuration presented in the following is unlikely to be the final Advanced Virgo configuration, but rather represents a snapshot of a development process. The numerical computations of the detector sensitivity have been performed with GWINC 42 34 .

In the following we compare the expected sensitivity for a configuration using the fundamental $\mathrm{TEM}_{00}$ mode against a configuration using a $\mathrm{LG}_{33}$ mode in three different scenarios in which we assumed a fixed mirror radius of $r_{\text {mirror }}=0.17 \mathrm{~m}$ [4] . The three scenarios are:

1. The beams of both mode configurations experience an identical clipping loss at the ITM/ETM mirrors. Hence, the beam size used in each configuration is different which results in a different $\mathrm{RoC}$ of the arm cavity mirrors. (see Sec. II). This means that for a change of the mode shape used in the interferometer from e.g. initially the $\mathrm{TEM}_{00}$ mode to the $\mathrm{LG}_{33}$ mode, all interferometer mirrors have to be exchanged.

2. Both mode configurations use arm cavity mirrors with an identical RoC. This enables a simple switching between the two different mode shapes.

3. The planned thermal compensation system (TCS) is used to change the RoC of the arm cavity mirrors by a certain amount. Hence one can start with one mode configuration which uses arm cavity mirrors with the optimal RoC. Later the TCS system 
enables us to change the RoC to optimise the clipping loss of the other mode configuration. Hence, this scenario is divided into two parts. The first one starts with an optimised parameter set for the TEM $_{00}$ mode and the TCS system is used to implement the possible $\mathrm{LG}_{33}$ mode configuration. In the second part of this scenario we will start with an optimised parameter set for the $\mathrm{LG}_{33}$ mode and than change to a $\mathrm{TEM}_{00}$ mode configuration using the TCS system.

The figures of merit of the comparison are the resulting effective detection range for a binary neutron star inspiral $\Gamma_{\mathrm{NS} / \mathrm{NS}}$ and effective detection range for a binary black hole inspiral $\Gamma_{\mathrm{BH} / \mathrm{BH}}$.

\section{A. Scenario (i): Identical clipping loss}

The two mode configurations which are compared in this scenario will be the reference configurations throughout the whole analysis because of their fixed clipping loss of $1 \mathrm{ppm}$. According to Section $\Pi$ the beam sizes of each configuration are different as well as the RoC of the interferometer mirrors. This scenario and its two configurations allow us to see the potential of using higher-order Laguerre Gauss modes in future GW detectors for a fixed mirror size.

Table IV comprises the input parameters used in the analysis as well as the resulting inspiral ranges. As one can see the analysis was performed for two different signal recycling (SR) detunings 35] $-750 \mathrm{~Hz}$ and $300 \mathrm{~Hz}-$ to emphasise that the improvements vary with the detuning. For both detunings the improvement using the $\mathrm{LG}_{33}$ mode is significant. The total mirror thermal noise is decreased by a factor of up to 1.68. According to our simulation this results in a relative improvement of the inspiral ranges by $20 \%-21 \%$ for a SR detuning of $750 \mathrm{~Hz}$ and $23 \%-28 \%$ for a SR detuning of $300 \mathrm{~Hz}$. Hence the potential event rate 44 of the Advanced Virgo detector can be increased by a factor of up to 2.1 by using the $\mathrm{LG}_{33}$ mode instead of the fundamental $\mathrm{TEM}_{00}$ mode.

\section{B. Scenario (ii): Identical RoCs}

Our second scenario uses arm cavity mirrors with a fixed $\mathrm{RoC}$ leading to the same beam size at the mirrors for both mode configurations. The advantage of this configuration is that it allows using either $\mathrm{TEM}_{00}$ or the $\mathrm{LG}_{33}$ mode without exchanging the interferometer mirrors. To achieve this with a reasonable sensitivity for both configurations, a tradeoff concerning the clipping loss has to be made, see Section III for more details. As a result the beam size of the $\mathrm{TEM}_{00}$ mode will be smaller compared to the reference configuration. On the one hand this results in much smaller clipping loss for this configurations, but on the other hand the clipping loss of the $\mathrm{LG}_{33}$ mode configuration will go down to an acceptable value as well. The input parameters and the results of the simulation analysis for this scenario are shown in Table $\mathrm{V}$. The comparison of these configurations shows that the inspiral ranges in the case of the $\mathrm{LG}_{33}$ mode configuration are greater by at least $35 \%$ and up to $53 \%$. Although this looks promising, these two configurations have to be compared with the reference configurations for the same SR detuning analysed in scenario (i). We then find that the $\mathrm{LG}_{33}$ configuration of scenario (ii) performs $0.1 \%$ better concerning the $\mathrm{BH} / \mathrm{BH}$ inspiral range but the NS/NS inspiral range is $11 \%$ worse because of its higher clipping loss of $l_{\text {clip }}=30 \mathrm{ppm}$ due to the slightly larger beam size. This results in a decreased intra-cavity power which lowers the sensitivity of the detector. The $\mathrm{TEM}_{00}$ configuration of scenario(ii) is also much less sensitive compared to the reference configurations because of the small beam size and the resulting higher thermal noise. The inspiral ranges of the $\mathrm{TEM}_{00}$ configuration decrease by approximately $22 \%$ in comparison to the reference configuration. The weak performance of the both configurations renders this scenario not very favourable to be implemented into Advanced Virgo.

\section{Scenario (iii): Use TCS to adapt RoCs}

Our third scenario combines the advantages of the two earlier scenarios - high performance and compatibility between the different mode configurations. We propose to use the TCS, which is an essential part of future GW detectors, to introduce a constant offset onto the RoC of the arm cavity mirrors. This technique has already been demonstrated in the GEO 600 detector to match the $\mathrm{RoC}$ of the two interferometer end mirrors [36. The basic idea of this approach is to start with one mode configuration with a clipping loss of $l_{\text {clip }}=1 \mathrm{ppm}$ at each arm cavity mirror. The TCS will then be used later to change the RoC of the arm cavity mirrors to optimise the useable beam width for the corresponding other transversal mode. The currently planned TCS [37] for second generation GW detectors are designed to introduce a RoC change of $\Delta R_{c}=-120 \mathrm{~m}$. The TCS uses a ring heater placed near the mirror substrate. The thermal radiation produced, is partly absorbed by the mirror substrate thus deforming its original shape. By placing the ring heater either in front or behind the mirror one can actually change the sign of the $\mathrm{RoC}$ change allowing an adjustment of $\Delta R_{c} \pm 120 \mathrm{~m}$. The two different signs of the possible RoC offset allow two different approaches whose input parameters and results are comprised in Table VI.

The first approach uses the reference $\mathrm{TEM}_{00}$ mode configuration with $l_{\text {clip }}=1 \mathrm{ppm}$ as initial interferometer configuration. This configuration with an arm cavity RoC of $R_{c}=1522.8 \mathrm{~m}$ has already been analysed in scenario (i). A constant change of the arm cavity RoC by $\Delta R_{c}=+120 \mathrm{~m}$ introduced by the TCS will minimise the 


\begin{tabular}{c|ccccc}
\hline \hline $\begin{array}{c}\text { Scenario } \\
(\mathrm{i})\end{array}$ & $\begin{array}{c}\text { SR det. } \\
{[\mathrm{Hz}]}\end{array}$ & $\begin{array}{c}w \text { in } \\
{[\mathrm{cm}]}\end{array}$ & $\begin{array}{c}l_{\text {clip }} \\
{[\mathrm{ppm}]}\end{array}$ & $\begin{array}{c}\text { RoC } \\
{[\mathrm{m}]}\end{array}$ & $\begin{array}{c}\Gamma_{\mathrm{NS} / \mathrm{NS}} \\
{[\mathrm{Mpc}]}\end{array}$ \\
\hline \hline TEM $_{00}$ & 750 & 6.47 & 1 & 1522.8 & 139.83 \\
$\mathrm{LG}_{33}$ & 750 & 3.94 & 1 & 1708.4 & 168.34 \\
$\mathrm{TEM}_{00}$ & 300 & 6.47 & 1 & 1522.8 & 148.85 \\
$\mathrm{LG}_{33}$ & 300 & 3.94 & 1 & 1708.4 & 191.26 \\
\hline \hline
\end{tabular}

Table IV: Input parameters and results of the GWINC simulation analysis of the scenario (i) which uses identical clipping loss at the arm cavity mirrors for each mode configuration.

\begin{tabular}{ccccccr}
\hline \hline $\begin{array}{c}\text { Scenario } \\
\text { (ii) }\end{array}$ & $\begin{array}{c}\text { SR det. } \\
{[\mathrm{Hz}]}\end{array}$ & $\begin{array}{c}w \text { in } \\
{[\mathrm{cm}]}\end{array}$ & $\begin{array}{c}l_{\text {clip }} \\
{[\mathrm{ppm}]}\end{array}$ & $\begin{array}{c}R_{c} \\
{[\mathrm{~m}]}\end{array}$ & $\begin{array}{r}\Gamma_{\mathrm{NS} / \mathrm{NS}} \\
{[\mathrm{Mpc}]}\end{array}$ & $\begin{array}{r}\Gamma_{\mathrm{BH} / \mathrm{BH}} \\
{[\mathrm{Mpc}]}\end{array}$ \\
\hline \hline $\mathrm{TEM}_{00}$ & 750 & 4.22 & $8.0 \mathrm{e}-9$ & 1647.2 & 110.86 & 900.47 \\
$\mathrm{LG}_{33}$ & 750 & 4.22 & 30 & 1647.2 & 149.25 & 1375.3 \\
\hline \hline
\end{tabular}

Table V: Input parameters and results of the GWINC simulation analysis of the scenario (ii) which uses mirrors with an identical RoC for each mode configuration.

beam size of a $\mathrm{LG}_{33}$ mode. This minimised mode still experiences a clipping loss of $l_{\text {clip }}=40.9 \mathrm{ppm}$. Please note that in this case the clipping losses of the $\mathrm{LG}_{33}$ mode will go down further with a larger RoC offset. The major advantage of this approach is that we can use the reference $\mathrm{TEM}_{00}$ mode configuration initially and than later exchange it for a better performing $\mathrm{LG}_{33}$ mode configuration. This is reflected in the resulting inspiral ranges of these two configurations (see TableVI). We find that the $\mathrm{NS} / \mathrm{NS}$ and the $\mathrm{BH} / \mathrm{BH}$ inspiral ranges of the $\mathrm{LG}_{33}$ mode configuration are increased by $2 \%$ and $18 \%$, compared to the reference $\mathrm{TEM}_{00}$ mode configuration. For a higher RoC change $\Delta R_{c}$ we can expect a much better performance due to the lower clipping loss. To reach the reference $\mathrm{LG}_{33}$ mode configuration from scenario (i) which has a clipping loss of $l_{\text {clip }}=1 \mathrm{ppm}$, requires for example an induced RoC change by the TCS of $\Delta R_{c}=186 \mathrm{~m}$.

The second approach uses the TCS to start with the reference $\mathrm{LG}_{33}$ mode configuration with $l_{\text {clip }}=1 \mathrm{ppm}$ and an arm cavity mirror RoC of $R_{c}=1708.4 \mathrm{~m}$ (see scenario (i)). The TCS can be used to introduce a constant offset in the arm cavity mirror $\mathrm{RoC}$ of $\Delta R_{c}=-120 \mathrm{~m}$, which maximises the possible beam size of a corresponding $\mathrm{TEM}_{00}$ mode configuration to $w=4.71 \mathrm{~cm}$ corresponding to the very small clipping loss $l_{\text {clip }}=4.8 \mathrm{e}-6 \mathrm{ppm}$. If the TCS is able to change the RoC by a larger amount, the usable beam size of the $\mathrm{TEM}_{00}$ mode configuration could be increased further. This approach leads to an increase of the inspiral ranges of the $\mathrm{LG}_{33}$ mode configuration by between $42 \%$ and $43 \%$ compared to the $\mathrm{TEM}_{00}$ mode configuration. Despite these large improvements with the $\mathrm{LG}_{33}$ mode configuration it is interesting to compare the $\mathrm{TEM}_{00}$ mode configuration used here to the reference $\mathrm{TEM}_{00}$ mode configuration used in scenario (i) to see how much one would lose by using this second approach of scenario (iii). It turns out that the inspiral ranges go down by approximately $15 \%$.
In conclusion scenario (iii) is the most promising one and the idea to use the TCS to introduce a constant change to the RoC of the arm cavity mirrors has great potential. It would allow a change from a $\mathrm{TEM}_{00}$ mode configuration to a $\mathrm{LG}_{33}$ mode configuration without exchanging the main optics of the interferometer. To decide which of the two approaches described is the better one, one has to judge if one could either afford to have clipping losses for the $\mathrm{LG}_{33}$ mode configuration of $l_{\text {clip }}=40.9 \mathrm{ppm}$ in the first approach, or to have a decreased performance of $15 \%$ by the $\mathrm{TEM}_{00}$ mode configuration in the second approach compared to the reference $\mathrm{TEM}_{00}$ mode configuration.

\section{Sensitivity improvement from the use of $\mathbf{L G}_{33}$ on the example of Advanced Virgo}

As an illustrating example we evaluate how much the sensitivity of an detector like Advanced Virgo could be improved by the application of $\mathrm{LG}_{33}$ modes. In particular we compare configurations featuring $\mathrm{TEM}_{00}$ and $\mathrm{LG}_{33}$ modes with identical clipping losses, but RoCs optimised for the individual modes. Our analysis made use of the same parameters as the most recent and comprehensive noise model for Advanced Virgo [39, 38. We assumed the following reduction factors for the $\mathrm{LG}_{33}$ mode in our GWINC simulation: The coating Brownian and the substrate Brownian noise are reduced by factors 1.7 and 1.945 , respectively, while the thermo-elastic noise increases by a factor 1.7 [40].

Figure 8 displays the resulting sensitivity curves (blue traces) of Advanced Virgo featuring TEM TE $_{00}$ (dashed line) and $\mathrm{LG}_{33}$ (solid line) modes. As coating Brownian noise is directly limiting the Advanced Virgo sensitivity in the frequency range between 40 and $200 \mathrm{~Hz}$, an impressive sensitivity improvement can be achieved by application 


\begin{tabular}{c||ccccc}
\hline \hline $\begin{array}{c}\text { Scenario } \\
\text { (iii) }\end{array}$ & $\begin{array}{c}\text { SR det. } \\
{[\mathrm{Hz}]}\end{array}$ & $\begin{array}{c}w \text { in } \\
{[\mathrm{cm}]}\end{array}$ & $\begin{array}{c}l_{\text {clip }} \\
{[\mathrm{ppm}]}\end{array}$ & $\begin{array}{c}R_{c} \\
{[\mathrm{~m}]}\end{array}$ & $\begin{array}{c}\Gamma_{\mathrm{NS} / \mathrm{NS}} \\
{[\mathrm{Mpc}]}\end{array}$ \\
\hline \hline TEM $_{00}$ & 750 & 6.47 & 1 & 1522.8 & 139.83 \\
LG $_{33}$ & 750 & 4.25 & 40.9 & 1642.2 & 142.93 \\
LG $_{33}$ & 750 & 3.94 & 1 & 1708.4 & 168.34 \\
TEM $_{00}$ & 750 & 4.71 & $4.8 \mathrm{e}-6$ & 1588.2 & 118.40 \\
\hline \hline
\end{tabular}

Table VI: Input parameters and results of the GWINC simulation analysis of the scenario (iii) which uses the same clipping loss at the arm cavity mirrors for each mode configuration.

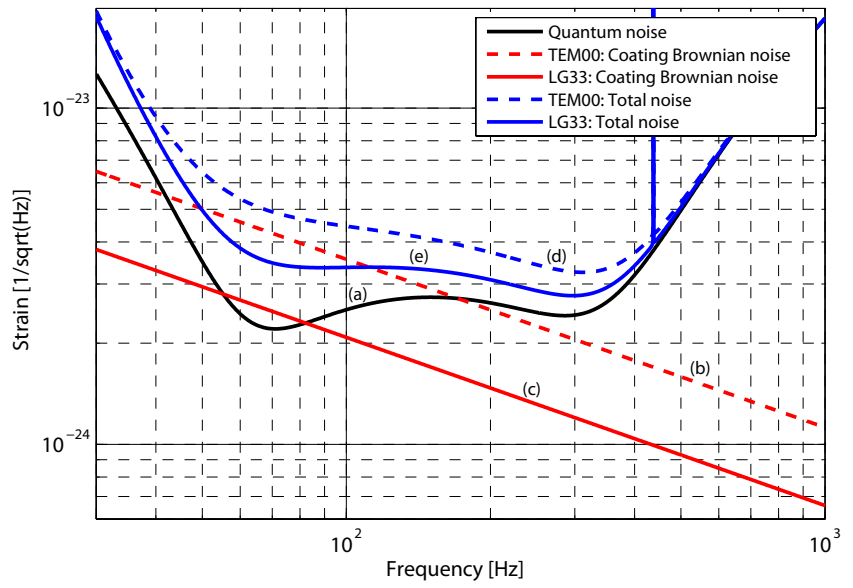

Figure 8: Sensitivity improvement from the implementation of the $\mathrm{LG}_{33}$ mode into the Advanced Virgo detector. This analysis is based on the detector configuration described in the Advanced Virgo Preliminary design [38. For simplicity only the contributions of coating Brownian noise (b, c) and quantum noise (a) are shown, while all other noise contributions are omitted in the plot, but taken into account for the overall sensitivity $(\mathrm{d}, \mathrm{e})$. The sensitivity improvement when going from $\mathrm{TEM}_{00}$ (d) to $\mathrm{LG}_{33}$ (e) mode corresponds to an improvement of the binary neutron star inspiral range from 145 to $185 \mathrm{Mpc}$ and increase the detectors NS/NS event rate by a factor of 2.1 .

of the $\mathrm{LG}_{33}$ mode. This broadband sensitivity improvement is concentrated on the range from 30 to $400 \mathrm{~Hz}$ with a maximal gain around $75 \mathrm{~Hz}$. The binary neutron star inspiral range increases from 145 to $185 \mathrm{Mpc}$, which corresponds to a rise of the expected NS/NS event rate by a factor of 2.1 .

\section{SUMMARY AND OUTLOOK}

We carried out a comprehensive analysis of the prospects of higher-order LG modes for future gravitational wave detectors. Using numerical interferometer simulations, we compared the behaviour of the $\mathrm{LG}_{33}$ mode with the fundamental mode $\left(\mathrm{TEM}_{00}\right)$. Our analysis included tilt to longitudinal phase coupling, generation of angular control signals and the corresponding control matrices for a single Fabry-Perot cavity as well as the coupling of differential arm cavity misalignment into dark port power for a full Michelson interferometer with arm cavities. We were able to show that the $\mathrm{LG}_{33}$ mode performs similar if not even better than the commonly used $\mathrm{TEM}_{00}$ for all considered aspects of interferometric sensing. This strongly indicates that all currently available experience and technology for interferometric sensing and control, which is based on the $\mathrm{TEM}_{00}$ mode, can be transferred to the use of the $\mathrm{LG}_{33}$ mode. Changing over from the fundamental mode to the $\mathrm{LG}_{33}$ will not require any fundamental changes of the interferometric control strategy or the control hardware, but only small adjustments of the involved parameters, such as servo gains.

In addition, we performed a quantitative evaluation of the expected sensitivity improvement from application of the $\mathrm{LG}_{33}$ mode, using the planned Advanced Virgo detector as an example. Three different options of how to change over from the $\mathrm{TEM}_{00}$ to the $\mathrm{LG}_{33}$ mode have been developed. The first scenario considers replacing the main mirrors, by ones with radii of curvature optimised for the $\mathrm{LG}_{33}$ mode, while the second scenario assumes that the same mirrors are used for both modes, resulting in different clipping losses. In the third scenario the Advanced Virgo thermal compensation system is used to adjust the mirror curvatures for the two optical modes of interest independently. The maximum sensitivity improvement is found to be achievable when replacing the mirrors (scenario (i)). Using the latest design parameters of Advanced Virgo we were able to show that the application of the $\mathrm{LG}_{33}$ mode can give a broadband improvement of the Advanced Virgo sensitivity for all frequencies in the range from 30 to $400 \mathrm{~Hz}$. The corresponding binary neutron star inspiral range increases from 145 to $185 \mathrm{Mpc}$, enhancing the potential detection rate of binary neutron star inspirals by a factor 2.1 .

The next steps towards the actual implementation of higher-order LG modes in future GW detectors have to include the demonstration of efficient generation of high power LG beams, followed by setting up of tabletop experiments for experimental verification of the simulations presented in the first half of this article. 


\section{ACKNOWLEDGEMENT}

We would like to thank J. Y. Vinet for fruitful discussions and for providing us with the thermal noise suppression factors for the higher-order LG modes. This work has been supported by the Science and Technology Facilities Council (STFC) and the European Gravitational
Observatory (EGO). This document has been assigned the LIGO Laboratory document number ligo-p0900006. v1.

\section{References}

[1] S. Hild and the LIGO Scientific Collaboration, Classical and Quantum Gravity 23, 643 (2006).

[2] F. Acernese and the VIRGO Collaboration, Classical and Quantum Gravity 23, 635 (2006).

[3] D. Sigg, Classical and Quantum Gravity 21, 409 (2004).

[4] R. Takahashi and the TAMA Collaboration, Classical and Quantum Gravity 21, 403 (2004).

[5] S. Hild, G. Losurdo, and A. Freise, Tech. Rep. VIR-101A08, Virgo (2008).

[6] K. Yamamoto, T. Uchiyama, S. Miyoki, M. Ohashi, K. Kuroda, H. Ishitsuka, T. Akutsu, S. Telada, T. Tomaru, T. Suzuki, et al., Journal of Physics: Conference Series 122, 012002 (8pp) (2008), URL http: //stacks.iop.org/1742-6596/122/012002

[7] M. Bondarescu and K. S. Thorne, Physical Review D (Particles, Fields, Gravitation, and Cosmology) 74, 082003 (pages 6) (2006), URL http://link.aps.org/ abstract/PRD/v74/e082003

[8] M. Bondarescu, O. Kogan, and Y. Chen, Physical Review D (Particles, Fields, Gravitation, and Cosmology) 78, 082002 (2008), URL http://link.aps.org/doi/10. 1103/PhysRevD.78.082002.

[9] B. Mours, E. Tournefier, and J.-Y. Vinet, Classical and Quantum Gravity 23, 5777 (2006).

[10] H. He, M. E. J. Friese, N. R. Heckenberg, and H. Rubinsztein-Dunlop, Phys. Rev. Lett. 75, 826 (1995).

[11] K. Bongs, S. Burger, S. Dettmer, D. Hellweg, J. Arlt, W. Ertmer, and K. Sengstock, Phys. Rev. A 63, 031602 (2001).

[12] J. Arlt, K. Dholakia, L. Allen, and M. J. Padgett, Journal of Modern Optics 45, 1231 (1998).

[13] M. A. Clifford, J. Arlt, J. Courtial, and K. Dholakia, Optics Communications 156, 300 (1998).

[14] S. A. Kennedy, M. J. Szabo, H. Teslow, J. Z. Porterfield, and E. R. Abraham, Phys. Rev. A 66, 043801 (2002).

[15] J. Courtial and M. J. Padgett, Optics Communications 159, 13 (1999).

[16] A. T. O'Neil and J. Courtial, Optics Communications 181, 35 (2000).

[17] S.-C. Chu and K. Otsuka, Optics Communications 281, 1647 (2008).

[18] A. Siegman, Lasers (University Science Books, 1986).

[19] Errata List for LASERS, http://www.stanford.edu/ siegman/lasers_book_errata.pdf.

[20] M. W. Beijersbergen, L. Allen, H. E. L. O. van der Veen, and J. P. Woerdman, Optics Communications 96, 123 (1993).

[21] Y. Levin, Phys. Rev. D 57, 659 (1998), arXiv:grqc/9707013.

[22] J.-Y. Vinet, to be published in Living Reviews in General Relativity, http://relativity.livingreviews.org/.
[23] A. Freise, G. Heinzel, H. Lück, R. Schilling, B. Willke, and K. Danzmann, Classical and Quantum Gravity 21, S1067 (2004), software available at: http://www.rzg.mpg.de/ adf, URL http://stacks .iop. org/0264-9381/21/S1067.

[24] A. Freise and M. Mantovani, Tech. Rep. VIR-NOT-EGO1390-330, Virgo (2006).

[25] J. A. Sidles and D. Sigg, Physics Letters A 354, 167 (2006).

[26] E. D. Black, Am. J. Phys. 69, 79 (2000).

[27] E. Morrison, D. I. Robertson, H. Ward, and B. J. Meers, Appl. Opt. 33, 5041 (1994).

[28] M. Mantovani and A. Freise, Journal of Physics Conference Series 122, 012026 (2008).

[29] M. Mantovani, Ph.D. thesis, Universita degli Studi di Siena (2007).

[30] Advanced LIGO, http://www.ligo.caltech.edu/advLIGO/.

[31] R. Adhikari, S. Ballmer, and P. Fritschel, Tech. Rep. LIGO-T070236-00-D, LIGO (2008).

[32] R. Abbott, R. Adhikari, S. Ballmer, L. Barsotti, M. Evans, P. Fritschel, V. Frolov, G. Mueller, B. Slagmolen, and S. Waldman, Tech. Rep. LIGO-T070247-00-I, LIGO (2008).

[33] Advanced VIRGO, http://wwwcascina.virgo.infn.it/advirgo/.

[34] GWINC, http://lhocds.ligowa.caltech.edu:8000/advligo/GWINC.

[35] S. Hild, H. Grote, M. Hewitson, H. Lück, J. R. Smith, K. A. Strain, B. Willke, and K. Danzmann, Classical and Quantum Gravity 24, 1513 (2007), URL http:// stacks.iop.org/0264-9381/24/1513

[36] H. Lück, A. Freise, S. Goßler, S. Hild, K. Kawabe, and K. Danzmann, Classical and Quantum Gravity 21, S985 (2004), URL http://stacks .iop.org/0264-9381/ 21/S985

[37] G. H. G. Billingsley and W. Kells, Tech. Rep. LIGOT080026-00-D, LIGO (2008).

[38] The Virgo Collaboration, Tech. Rep., Virgo (2008).

[39] S. Hild and G. Losurdo, Tech. Rep. VIR-055A-08, Virgo (2008).

[40] J.-Y. Vinet, personal communication.

[41] Please note that we use the equivalent terms fundamental mode, TEM $M_{00}$ mode, $H G_{00}$ mode and $L G_{00}$ mode throughout this paper, depending on which of these seems to be more appropriate in the context.

[42] In our analysis presented here we use a specifically to Advanced Virgo adapted version of the simulation program GWINC - Gravitational Wave Interferometer Noise Calculator.

[43] In principle the Virgo mirror radius is $r_{\text {mirror }}=0.175 \mathrm{~m}$, but a phase of $5 \mathrm{~mm}$ around the mirror limits the actual coating radius to $\rho=0.17 \mathrm{~m}$. Therefore the actual clip- 
ping loss of the reflected beam has to be calculated using this coating radius.

[44] Assuming an uniform distribution of sources in space, the event rate of a gravitational detector is proportional to the volume of space it can sense and therefore grows with the cube of the distance to a detectable source.

[45] These numbers can be calculated using the formulas for the coating and substrate Brownian thermal noise for finite sized mirrors 22 in conjunction with the follow- ing parameters. $w_{\mathrm{LG00}}=6.47 \mathrm{~cm} ; w_{\mathrm{LG} 33}=3.94 \mathrm{~cm}$; $r_{\text {mirror }}=0.17 \mathrm{~m} ;$ mirror thickness $h=0.2 \mathrm{~m} ; \lambda=$ $1064 \mathrm{~nm} ; f=1 \mathrm{~Hz} ; T=300 \mathrm{~K}$; number of $\mathrm{SiO}_{2}$ coating layers $N_{\mathrm{SiO}_{2}}=21$; number of $\mathrm{Ta}_{2} \mathrm{O}_{5}$ coating layers $N_{\mathrm{Ta}_{2} \mathrm{O}_{5}}=20 ; \phi_{\text {substrate }}=5 e^{-9} ; \phi_{\mathrm{SiO}_{2}}=5 e^{-5}$; $\phi_{\mathrm{Ta}_{2} \mathrm{O}_{5}}=2 e^{-4} ; \sigma_{\text {substrate }}=0.167 ; Y_{\text {substrate }}=72.7 \mathrm{GPa}$; refractive indices $n_{\mathrm{SiO}_{2}}=1.44876$ and $n_{\mathrm{Ta}_{2} \mathrm{O}_{5}}=2.06$ 